\title{
Spectral triples and the geometry of fractals
}

\author{
Erik Christensen, Cristina Ivan, and Elmar Schrohe
}

\begin{abstract}
We construct spectral triples for the Sierpinski gasket as infinite sums of unbounded Fredholm modules associated with the holes in the gasket and investigate their properties. For each element in the K-homology group we find a representative induced by one of our spectral triples. Not all of these triples, however, will have the right geometric properties. If we want the metric induced by the spectral triple to give the geodesic distance, then we will have to include a certain minimal family of unbounded Fredholm modules. If we want the eigenvalues of the associated generalized Dirac operator to have the right summability properties, then we get limitations on the number of summands that can be included. If we want the Dixmier trace of the spectral triple to coincide with a multiple of the Hausdorff measure, then we must impose conditions on the distribution of the summands over the gasket. For the elements of a large subclass of the K-homology group, however, the representatives are induced by triples having the desired geometric properties. We finally show that the same techniques can be applied to the Sierpinski pyramid.
\end{abstract}

Mathematics Subject Classification (2010). Primary 28A80, 46L87; Secondary 53C22, 58B34. Keywords. Spectral triple, non commutative geometry, K-homology, Sierpinski gasket.

\section{Introduction}

In his noncommutative geometry program Alain Connes employs ideas from operator algebras to analyze singular spaces for which the classical tools of geometric analysis fail. One of the basic structures in this theory is that of a spectral triple $(\mathcal{A}, \mathscr{H}, D)$, consisting of an algebra $\mathcal{A}$ of bounded operators on a Hilbert space $\mathscr{H}$ and an unbounded selfadjoint operator $D$ on $\mathscr{H}$. In this picture, the space is replaced by the algebra $\mathcal{A}$ - in the simplest cases an algebra of sufficiently smooth functions on the space -, while the geometry is encoded in the operator $D$, which is required to have a compact resolvent and bounded commutators with the elements of $\mathcal{A}$. In [Co2], [Co4] Connes proves with some relevant examples that his program may be used to study fractals. In [La1], [La2] Michel Lapidus investigates in many different ways the possibility of developing a noncommutative fractal geometry. In [CI] Christensen and Ivan construct spectral triples for approximately finite dimensional $\mathrm{C}^{*}$-algebras and then apply this result to the special case of the continuous functions on the Cantor set. In [CIL] Christensen, Ivan and Lapidus construct a spectral triple 
associated to the Sierpinski gasket. It encodes the geometry in that it recovers the geodesic distance, the Hausdorff dimension and the Hausdorff measure; moreover, it gives a non-trivial element in the K-homology group of the gasket.

In this paper, our main interest is to determine which K-homology elements we can obtain by constructions of this type. At the same time, we extend the analysis to the case of the Sierpinski pyramid. In both cases, a crucial role is played by the sets HSG and HSP of holes in the gasket and non-horizontal holes in the pyramid, respectively. In fact, the $K_{1}$ groups are just the free abelian groups

$$
\bigoplus_{\mathrm{HSG}} \mathbb{Z} \text { and } \bigoplus_{\mathrm{HSP}} \mathbb{Z}
$$

and the K-homology groups $K^{1}$ are the dual groups with respect to $\mathbb{Z}$,

$$
\prod_{\mathrm{HSG}} \mathbb{Z} \text { and } \prod_{\mathrm{HSP}} \mathbb{Z}
$$

A K-homology element can therefore be identified with a sequence of integers indexed by HSG and HSP, respectively.

We show that it is possible to obtain any element in the K-homology group from our construction, but the only geometric structure which is preserved by all such spectral triples is the geodesic distance. If we want to have a spectral triple of this type which gives the right Hausdorff dimension, then there is some limitation on the growth of the associated sequence representing the K-homology element. Whenever this sequence is bounded, we find a spectral triple with the right metric and the right dimension. There is room for some unbounded sequences too, but we are not able to say exactly which ones we can obtain. The Hausdorff measure is dominated by the volume measure induced by any of our spectral triples. In general, however, the two measures are not proportional. We find sufficient conditions for this to be true. These investigations show that inside our set-up there are bounds on the numbers as well as on the distributions of summands we use in forming our spectral triples. In the other direction we can prove that the spectral triple, which we denote ZGT, and which represents the 0 -element in the K-homology group, actually has a minimality property. We do this by showing that if just any summand - in the sum of unbounded Fredholm modules giving this spectral triple - is left out, then the geodesic distance will not be the metric coming from this spectral triple.

Acknowledgment. We are grateful to Malek Joumaah for the drawings.

\section{Sierpinksi gasket: constructions, K-theory and K-homology}

There are two basic procedures which both produce the Sierpinski gasket; one is based on continued cuttings and the other on continued extensions of graphs. The first steps in the cutting procedure are shown in the Figure 1 below. 

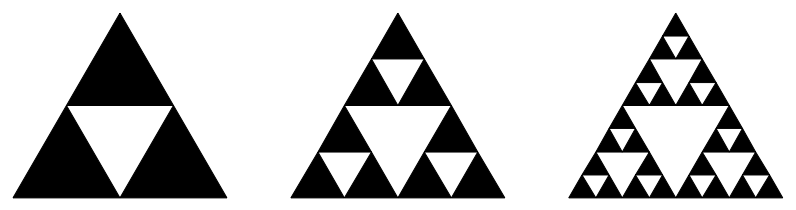

Figure 1

The Sierpinski gasket is just the intersection of all these sets, and a compactness argument shows that this is a non-empty compact subset of the plane. The inductive construction procedure shows that there are many holes, i.e., bounded components of the complement of the gasket, and once a hole has been added it will remain undisturbed during the following steps of the construction.

The first steps in the extension procedure are shown in the Figure 2 below.
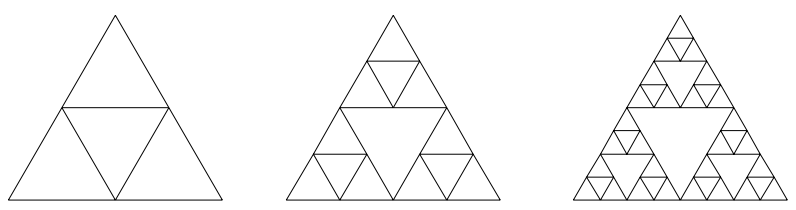

Figure 2

In this case the gasket is obtained as the closure of the union of all these sets, which may be thought of as planar graphs.

The $K_{1}$ and the first Steenrod K-homology group $K^{1}$ of the gasket are computable from general results on the K-theory and K-homology of planar sets. Let $X$ be a nonempty compact Hausdorff space and $\breve{H}^{1}(X)$ the first Čech cohomology group of $X$, i.e., $\breve{H}^{1}(X)$ is the quotient of the multiplicative group $C(X)^{-1}$ of continuous non-vanishing functions on $X$, by its component of unity. Define a character homomorphism $K_{1}(C(X)) \rightarrow \breve{H}^{1}(X)$ by assigning to a unitary in $M_{k}(C(X))-$ which is a continuous, unitary-valued function on $X$ - the class of the function which is obtained as the pointwise determinant. If $X$ is a non-empty, compact subset of the plane then this is an isomorphism (see for example [HR]).

The following description of $\breve{H}^{1}(X)$ makes it clear that $K_{1}(C(X))$ is free abelian.

Theorem 1.1 ([HR]). Let $\left\{\lambda_{1}, \lambda_{2}, \ldots\right\}$ be a sequence of points in $\mathbb{C} \backslash X$ with precisely one $\lambda_{j}$ in each hole, i.e., bounded component of the complement of $X$, and none in the unbounded component of the complement. The group $\breve{H}^{1}(X)$ is freely generated 
as an abelian group by the homotopy classes of the nowhere zero complex functions $z-\lambda_{j}$ on $X$.

For the first Steenrod K-homology group of $X, K^{1}(C(X))$, there are two equivalent descriptions. The first is due to Brown, Douglas, Fillmore (see [BDF]) who realize $K^{1}(C(X))$ as the abelian group of unitarily equivalent extensions of $\mathrm{C}^{*}$-algebras of the form

$$
0 \rightarrow \mathcal{K} \rightarrow A \rightarrow C(X) \rightarrow 0,
$$

where $\mathcal{K}$ is the $\mathrm{C}^{*}$-algebra of compact operators on a separable complex Hilbert space. The second description is due to Kasparov [Ka2] (see also [Co1], [Co2]) who realizes $K^{1}(C(X))$ as the abelian group of stable homotopy classes of bounded Fredholm modules over $C(X)$.

If $X$ is a compact planar set

$$
K^{1}(C(X)) \cong \operatorname{Hom}\left(K_{1}(C(X), \mathbb{Z}) \quad([\mathrm{BDF}],[\mathrm{Do}])\right.
$$

In Kasparov's picture of $K^{1}$ ([Ka1], [Co2]) the isomorphism between $K^{1}(C(X))$ and $\operatorname{Hom}\left(K_{1}(C(X), \mathbb{Z})\right.$ is given by the following index map:

Let $(H(\pi), F)$ be an (odd bounded) Fredholm module over $C(X)$ and let $P=$ $(I+F) / 2$. Let $u$ be a unitary in $C(X)$. Then the operator $P \pi(u) P$ from $P H$ to itself is a Fredholm operator. An additive map from $K_{1}(C(X))$ to $\mathbb{Z}$ is determined by

$$
\Phi_{(H, F)}([u])=-\operatorname{Index}(P \pi(u) \mid P H) .
$$

The index map $\Phi_{(H, F)}$ only depends upon the class of $(H, F)$.

Baaj and Julg have shown in [BJ] that every class of bounded Fredholm modules over the algebra $C(X)$ contains a Fredholm module constructed from an unbounded one (see also [Co2], IV, Appendix A). Precisely, any unbounded Fredholm module $(H, D)$ associated to $C(X)$ defines a bounded Fredholm module $(H, F)$, where $F$ is the self-adjoint unitary coming from the polar decomposition of the Dirac operator $D$. The symmetry $F$ equals $2 P-I$ where $P$ is the spectral projection $E([0, \infty[)$ for $D$. Every bounded Fredholm module over $C(X)$ is operator homotopic to one obtained from the foregoing construction.

Remark. The above description of $K_{1}(C(X))$ for any compact planar set $X$ identifies $\operatorname{Hom}\left(K_{1}(C(\mathrm{SG})), \mathbb{Z}\right)$, and thus $K^{1}(C(\mathrm{SG}))$, with the product group $\prod \mathbb{Z}$, with one factor for each hole in $\mathrm{SG}$.

\section{Some notation and conventions}

The basis for our constructions is Section 8 of [CIL], and we assume some familiarity with these results. There a spectral triple for the Sierpinski gasket is constructed as an example. We will call this triple the old gasket triple. Let us briefly recapture 
some of the definitions and names. The basis for the construction is an equilateral triangle of circumference $2 \pi$ which is placed as in Figure 2 such that one vertex is pointing upwards. This triangle is denoted by $\Delta_{0,1}$, and for any natural number $n$ the gasket contains $3^{n}$ triangles $\Delta_{n, j}, 1 \leq j \leq 3^{n}$, of circumference $2 \pi /\left(2^{n}\right)$ which are all scaled and translated copies of $\Delta_{0,1}$ as shown in Figure 2. Each of the triangles $\Delta_{n, j}$ is then treated as a circle with radius $2^{-n}$. In [CIL], Theorem 2.4 , we investigated the standard spectral triple for a circle of radius $2^{-n}$, and we showed how this triple can be transformed into an unbounded Fredholm module for $C(\mathrm{SG})$. We will let $\operatorname{UFM}\left(\Delta_{n, j}\right)$ denote this unbounded Fredholm module. The direct sum of these unbounded Fredholm modules over all the pairs $(n, j)$ then gives the old spectral triple. Later, the authors discussed other possibilities for a spectral triple for the Sierpinski gasket. It is clear that the upside down triangles which form the boundaries of the bounded components of the complement of the gasket must contain nearly the same information as the triangles $\Delta_{n, j}$. The triangle $\Delta_{0,1}$ then only will appear indirectly as a boundary in a completion of the union of all the upsidedown triangles, but this is not a serious problem, since we can just add the module corresponding to the outer triangle to the direct sum of the modules associated to all the upside down triangles. To be more precise we will also introduce a numbering system for the upside down triangles. The central one is denoted by $\nabla_{1,1}$; it is of circumference $\pi$. All the upside down triangles are then numbered by a pair $(m, k)$ with $m, k$ natural numbers such that $1 \leq k \leq 3^{m-1}$, and they are denoted by $\nabla_{m, k}$.

With HSG denoting the countable set that enumerates the holes in the Sierpinski gasket, i.e.,

$$
\mathrm{HSG}:=\left\{(m, k) \mid m \in \mathbb{N}, 1 \leq k \leq 3^{m-1}\right\},
$$

we may write

$$
\begin{aligned}
& K_{1}(C(\mathrm{SG}))=\bigoplus_{(m, k) \in \mathrm{HSG}} \mathbb{Z}, \\
& K^{1}(C(\mathrm{SG}))=\operatorname{Hom}\left(K_{1}(C(\mathrm{SG})), \mathbb{Z}\right)=\prod_{(m, k) \in \mathrm{HSG}} \mathbb{Z} .
\end{aligned}
$$

\section{On a family of spectral triples representing any element in $K^{1}(C(\mathrm{SG}))$}

Just as for the triangles $\Delta_{n, j}$ we can construct an unbounded Fredholm module,

$$
\operatorname{UFM}\left(\nabla_{m, k}\right)=\left(A, H_{\nabla_{m, k}}, D_{\nabla_{m, k}}\right),
$$

for the Sierpinski gasket by parameterizing $\nabla_{m, k}$ on a circle of radius $2^{-m}$ following [CIL], Definition 8.1. For each of the triangles $\Delta_{n, j}$, respectively $\nabla_{m, k}$, we can also construct an unbounded Fredholm module by reversing the orientation in the parameterization of the triangles. These modules will be denoted by $\overline{\operatorname{UFM}\left(\Delta_{n, j}\right)}$ and $\overline{\mathrm{UFM}}\left(\nabla_{m, k}\right)$, respectively. 
Proposition 3.1. Let $u$ be a unitary in $C(\mathrm{SG}),[u]$ its class in $K_{1}(C(\mathrm{SG}))$. For each of the triangles $\Delta_{n, j}$, respectively $\nabla_{m, k}$, denote the winding number for the restriction of $u(z)$ to this triangle by $w_{\Delta_{n, j}}(u)$, respectively $w_{\nabla_{m, k}}(u)$. Then:

(i) $\Phi_{\mathrm{UFM}\left(\Delta_{n, j}\right)}([u])=w_{\Delta_{n, j}}(u)$.

(ii) $\Phi_{\mathrm{UFM}\left(\nabla_{m, k}\right)}([u])=w_{\nabla_{m, k}}(u)$.

(iii) $\Phi_{\mathrm{UFM}\left(\Delta_{n, j}\right)}([u])=\sum_{\nabla_{m, k} \subset \Delta_{n, j}} w_{\nabla_{m, k}}(u)$.

(iv) The element $\Phi_{\mathrm{UFM}\left(\Delta_{n, j}\right)}$ in the group

$$
\operatorname{Hom}\left(K_{1}(\mathrm{SG}), \mathbb{Z}\right)=\prod_{(m, k) \in \mathrm{HSG}} \mathbb{Z}
$$

is the function $f_{\Delta_{n, j}}: \mathrm{HSG} \rightarrow \mathbb{Z}$ given by

$$
f_{\Delta_{n, j}}(m, k)= \begin{cases}1 & \text { if } \nabla_{m, k} \subset \Delta_{n, j}, \\ 0 & \text { else. }\end{cases}
$$

Proof. (i) and (ii) are consequences of the result that for the unit circle $\mathbb{T}$, the Hilbert space $H=L^{2}(\mathbb{T})$ and the projection $P_{+}$onto $H_{+}=\overline{\operatorname{span}}\left\{z^{n} \mid n \geq 0\right\}$, the winding number of $u$ on $\mathbb{T}$ is the opposite integer to the index of $P_{+} M_{u} \mid H_{+}$, where $M_{u}$ is the multiplication operator induced by $u$.

For (iii) use the uniform continuity of $u$ : From a certain natural number $n_{0}$ on we have for any $n, m \geq n_{0}$ that the winding numbers of $u$ around any $\Delta_{n, j}$ and any $\nabla_{m, k}$ vanish for $m, n \geq n_{0}$. Let us next compute $\Phi_{\mathrm{UFM}\left(\Delta_{0,1}\right)}([u])$. By the same argument as above, it equals the winding number of $u$ around $\Delta_{0,1}$. On the other hand this is the sum of the winding numbers over the four triangles $\Delta_{1, j}, 1 \leq j \leq 3$, and $\nabla_{1,1}$. For each of $\Delta_{1, j}$ we repeat the subdivision until we reach the level $n_{0}$ from where on all winding numbers of $u$ vanish. We are left with the sum of all the winding numbers of $u$ over the triangles $\nabla_{m, k}, 1 \leq m \leq n_{0}, 1 \leq k \leq 3^{m-1}$, and obtain (iii) in the case where $n=0$ and $j=1$. The general case then follows by an analogous argument applied to the triangle $\Delta_{n, j}$.

(iv) is just a reformulation of (iii) which is suitable for the computations to come.

We recall from [CIL] that we can perform infinite-direct sums of unbounded Fredholm modules of the types

$$
\operatorname{UFM}\left(\nabla_{m, k}\right) \text { or } \overline{\operatorname{UFM}\left(\nabla_{m, k}\right)}
$$

and get an unbounded Fredholm module as a result.

We will now construct a spectral triple ZGT for the Sierpinski gasket which induces the trivial element of the group $K^{1}(C(\mathrm{SG}))$. This may seem a bit strange, but the idea is that ZGT - an acronym for Zero Gasket Triple - will carry all the geometric information on the geodesic distance, the Hausdorff dimension, and the 
volume form, but induce the zero-element in the K-homology group. If we then add any unbounded Fredholm module by direct sum to ZGT, the $K^{1}$ element induced by the sum equals that of the added unbounded Fredholm module.

The spectral triple ZGT is defined in very much the same way as the old gasket triple mentioned in Section 2. It is the direct sum of the unbounded Fredholm modules associated to all the upside down triangles $\nabla_{m, k}$ plus the unbounded Fredholm module coming from the outer triangle $\Delta_{0,1}$, with the orientation reversed:

Proposition 3.2. The direct sum of unbounded Fredholm modules

$$
\mathrm{ZGT}:=\overline{\operatorname{UFM}\left(\Delta_{0,1}\right)} \oplus\left(\bigoplus_{m=1}^{\infty}\left(\bigoplus_{k=1}^{3^{m-1}} \operatorname{UFM}\left(\nabla_{m, k}\right)\right)\right)
$$

is a spectral triple. The Hilbert space is denoted by $H_{\mathrm{ZGT}}$, the representation of $C(\mathrm{SG})$ on $H_{\mathrm{ZGT}}$ and the Dirac operator on $H_{\mathrm{ZGT}}$ are named $\pi_{\mathrm{ZGT}}$ and $D_{\mathrm{ZGT}}$. The bounded Fredholm module coming from the polar decomposition of $D_{\mathrm{ZGT}}$ induces the trivial element of the group $K^{1}(C(\mathrm{SG}))$.

Proof. The arguments from [CIL], pages 27-28, may be copied and show that the direct sum is a spectral triple.

The results (ii) and (iii) in Proposition 3.1 show that the corresponding element in $K^{1}(C(\mathrm{SG}))$ is trivial.

Theorem 3.3. The spectral triple ZGT has the following geometric properties:

(i) The metric induced by the ZGT is the geodesic distance.

(ii) The ZGT is summable for any positive $s>\log 3 / \log 2$. Its zeta-function $\zeta_{\mathrm{ZGT}}(s)$ is meromorphic with a simple pole at $\log 3 / \log 2$ and it is given by $\zeta_{\mathrm{ZGT}}(s)=$ $2 \frac{\left(2^{s}-1\right)\left(2^{s}-2\right)}{2^{s}-3} \cdot \zeta(s)$.

(iii) Let $\mu$ denote the Hausdorff probability measure of dimension $\log 3 / \log 2$ on the Sierpinski gasket. Then for any Dixmier trace and any continuous function $g$ in $C(\mathrm{SG})$ we have

$$
\operatorname{Tr}_{\omega}\left(\left|D_{\mathrm{ZGT}}\right|^{-\frac{\log 3}{\log 2}} \pi_{\mathrm{ZGT}}(g)\right)=\frac{4}{3 \log 3} \cdot \zeta\left(\frac{\log 3}{\log 2}\right) \cdot \int_{S G} g(x) d \mu(x) .
$$

Proof. Except for the computation of the zeta-function, the proof here can be copied from [CIL], Theorem 8.4, Proposition 8.6. With respect to the zeta-function, we get for any fixed natural number $l$ that the zeta function for $\Delta_{l, j}$ or $\nabla_{l, j}$ in a point $s>1$ equals

$$
\begin{aligned}
\sum_{k \in \mathbb{Z}} 2^{-l s}|k+1 / 2|^{-s} & =2 \cdot 2^{-l s} \cdot 2^{s} \sum_{k \in \mathbb{N}}(2 k+1)^{-s} \\
& =2 \cdot 2^{-l s} \cdot 2^{s} \cdot\left(1-2^{-s}\right) \cdot \zeta(s) \\
& =2^{1-l s} \cdot\left(2^{s}-1\right) \cdot \zeta(s) .
\end{aligned}
$$


Since there are $3^{m-1}$ triangles of the form $\nabla_{m, j}$ and just one of the form $\Delta_{0,1}$ we get that the zeta function for this spectral triple - for $s>\log (3) / \log (2)-$ is given by

$$
\begin{aligned}
\zeta_{\mathrm{ZGT}}(s) & =2\left(2^{s}-1\right) \cdot \zeta(s)+\sum_{m=1}^{\infty} 3^{m-1} \cdot 2 \cdot 2^{-m s}\left(2^{s}-1\right) \cdot \zeta(s) \\
& =2\left(2^{s}-1\right) \zeta(s)+2 \cdot\left(2^{s}-1\right) 2^{-s} \frac{1}{1-\left(3 / 2^{s}\right)} \cdot \zeta(s) \\
& =2 \frac{\left(2^{s}-1\right)\left(2^{s}-2\right)}{2^{s}-3} \cdot \zeta(s) .
\end{aligned}
$$

Further on, by Proposition 4 on page 306 in [Co2], we obtain

$$
\begin{aligned}
\operatorname{Tr}_{\omega}\left(\left|D_{\mathrm{ZGT}}\right|^{-\frac{\log 3}{\log 2}}\right) & =\lim _{x \rightarrow 1+}(x-1) \operatorname{Tr}\left(\left|D_{\mathrm{ZGT}}\right|^{-x \cdot \frac{\log 3}{\log 2}}\right) \\
& =\lim _{x \rightarrow 1+}(x-1) \zeta_{\mathrm{ZGT}}\left(x \cdot \frac{\log 3}{\log 2}\right) \\
& =2 \lim _{x \rightarrow 1+}(x-1) \frac{\left(2^{x \frac{\log 3}{\log 2}}-1\right)\left(2^{x \frac{\log 3}{\log 2}}-2\right)}{2^{x \frac{\log 3}{\log 2}}-3} \zeta\left(x \frac{\log 3}{\log 2}\right) \\
& =\frac{4}{3} \cdot \zeta\left(\frac{\log 3}{\log 2}\right) \cdot \lim _{x \rightarrow 1+} \frac{x-1}{3^{x-1}-1} \\
& =\frac{4}{3 \log 3} \cdot \zeta\left(\frac{\log 3}{\log 2}\right) .
\end{aligned}
$$

Definition 3.4. For $(f(m, k))_{m, k} \in \prod_{(m, k) \in \mathrm{HSG}} \mathbb{Z}$ let $\operatorname{UFM}(f)$ denote the direct sum

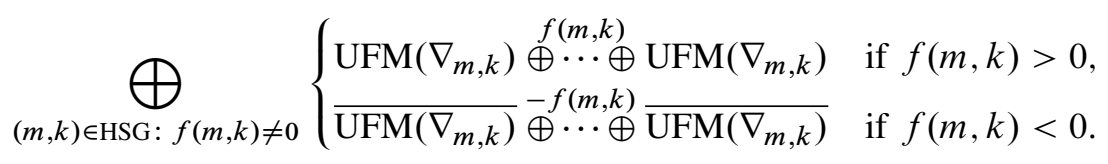

It is now quite easy to construct a spectral triple which induces any prescribed element in the group $K^{1}(C(\mathrm{SG}))$.

Definition 3.5. Let $(f(m, k))_{m, k} \in \prod_{(m, k) \in \mathrm{HSG}} \mathbb{Z}$ then $\mathrm{ST}(f)$ denotes the direct sum

$$
\mathrm{ZGT} \oplus \operatorname{UFM}(f) \text {. }
$$

Theorem 3.6. Let $(f(m, k))_{m, k} \in \prod_{(m, k) \in \mathrm{HSG}} \mathbb{Z}$. Then $\mathrm{ST}(f)$ is a spectral triple which induces the geodesic distance on the gasket and the $K^{1}$-element $(f(m, k))_{m, k}$.

Such a spectral triple will in general not have the right summability properties. In the first place the authors thought that the elements in $K^{1}(C(\mathrm{SG}))$ coming from spectral triples that encode the fractal geometry of the gasket ought to be a subgroup 
of $\operatorname{Hom}\left(K_{1}(C(\mathrm{SG})), \mathbb{Z}\right)$, but it is by no means clear or even true that the direct sum of two arbitrary unbounded Fredholm modules will make sense as an unbounded Fredholm module. The problem is analogous to the one coming from the addition of unbounded operators, namely that for two spectral triples $\left(A_{1}, H_{1}, D_{1}\right)$ and $\left(A_{2}, H_{2}, D_{2}\right)$ associated to a $C^{*}$-algebra $\mathcal{A}$ the intersection $A_{1} \cap A_{2}$ may be too small to be a dense subalgebra of $\mathcal{A}$.

This problem does not arise when forming the sum used in the definition of ST $(f)$, since the algebra $A$ is the same for all summands. We infer from the proof of Theorem 8.2 in [CIL] that $A$ is the algebra generated by the real affine functions on the plane, restricted to the gasket. Since ZGT is a summand in any ST $(f)$ it follows that the metric induced by the ST $(f)$ must be the one induced by ZGT which equals the geodesic distance on the gasket.

With respect to summability properties, it follows from the fact that ZGT is a direct summand in $\mathrm{ST}(f)$ that $\mathrm{ST}(f)$ can only be summable for $p>\log 3 / \log 2$. The zeta function for $\mathrm{ST}(f)$, say $\zeta_{f}$, is easily computed to be

$$
\begin{aligned}
\zeta_{f}(s) & =2 \frac{\left(2^{s}-1\right)\left(2^{s}-2\right)}{2^{s}-3} \cdot \zeta(s)+\sum_{m=1}^{\infty} \sum_{k=1}^{3^{m-1}}|f(m, k)|\left(2 \cdot 2^{-m s} \cdot\left(2^{s}-1\right) \cdot \zeta(s)\right) \\
& =\zeta_{\mathrm{ZGT}}(s)+2 \cdot\left(2^{s}-1\right) \zeta(s)\left(\sum_{m=1}^{\infty} \sum_{k=1}^{3^{m-1}}|f(m, k)| \cdot 2^{-m s}\right) .
\end{aligned}
$$

By applying the root criterion we see that the following result holds.

Theorem 3.7. Let $(f(m, k)) \in \prod_{\mathrm{HSG}} \mathbb{Z}$. Then $\mathrm{ST}(f)$ is summable for any $p>$ $\log 3 / \log 2$ if and only if

$$
\limsup _{m \rightarrow \infty}\left(\sum_{k=1}^{3^{m-1}}|f(m, k)|\right)^{1 / m} \leq 3 .
$$

There is an immediate corollary.

Corollary 3.8. If $\sup _{m, k}|f(m, k)|<\infty$, then $\mathrm{ST}(f)$ is summable for any $p>$ $\log 3 / \log 2$.

This result implies that the elements of the subgroup of the K-homology group $K^{1}$ given by bounded sequences are all represented by spectral triples with the right summability properties. We have thought of a possible converse to Theorem 3.7, and we conjecture that the following is true.

Conjecture 3.9. Let $(A, H, D)$ denote a spectral triple for $C(\mathrm{SG})$ which induces the geodesic distance on the gasket. If it is summable for any $p>\log 3 / \log 2$ and $(f(m, k)) \in \prod_{\mathrm{HSG}} \mathbb{Z}$ represents its $K$-homology class then

$$
\limsup _{m \rightarrow \infty}\left(\sum_{k=1}^{3^{m-1}}|f(m, k)|\right)^{1 / m} \leq 3 .
$$


At the time of the publication of this article we had no new information on the status of the conjecture.

The volume form obtained from a spectral triple $\mathrm{ST}(f)$ which is summable for $p>\log 3 / \log 2$ clearly majorizes a multiple of the one obtained from the $\log 3 / \log 2-$ dimensional Hausdorff measure, since ST $(f)$ contains ZGT as a direct summand. On the other hand it is rather obvious that even for a bounded sequence $(f(m, k))$ the values may be unevenly distributed so that the resulting volume form is not proportional to the one coming from the $\log 3 / \log 2$-dimensional Hausdorff measure. Below, we discuss conditions on $(f(m, k))$ that assure that the volume form given by $\operatorname{ST}(f)$ and the $\log 3 / \log 2$-dimensional Hausdorff measure are multiples of each other. We first give a definition, then state our results.

Definition 3.10. The sequence $(f(m, k))$ is said to be boundedly almost invariant, if the sequence of averages

$$
a(m):=\sum_{k=1}^{3^{m-1}} \frac{|f(m, k)|}{3^{m-1}}
$$

is bounded and

$$
\limsup _{m \rightarrow \infty}\left(\sum_{k=1}^{3^{m-1}}|| f(m, k)|-a(m)|\right)^{1 / m}<3
$$

Let $D_{f}$ and $\left(H_{f}, \pi_{f}\right)$ denote the Dirac operator and the representation of the spectral triple $\mathrm{ST}(f)$, respectively.

Lemma 3.11. Let $(f(m, k))$ be a boundedly almost invariant sequence. Then

$$
\left|D_{f}\right|^{-\log 3 / \log 2} \in \mathscr{L}^{(1, \infty)}\left(H_{f}\right) .
$$

(See beginning of [Co2], IV.2. $\beta$, p. 303).

Proof. According to our description of the spectral triple ST $(f)$ and the statement in front of Theorem 3.7 it follows that the $\zeta$-function for $s>\log 3 / \log 2$ is given as the infinite sum of non-negative values

$$
\operatorname{Tr}\left(\left|D_{f}\right|^{-s}\right)=\zeta_{f}(s)=\zeta_{\mathrm{ZGT}}(s)+2\left(2^{s}-1\right) \zeta(s)\left(\sum_{m=1}^{\infty} \sum_{k=1}^{3^{m-1}}|f(m, k)| \cdot 2^{-m s}\right)
$$

The theory we will use to prove the lemma is all based on limits as $x \rightarrow 1+$ rather than $s \rightarrow \log 3 / \log 2$, so we will replace the $\zeta$-function with the positive function $T(x)$ defined for $x>1$ by

$$
T(x):=\zeta_{f}\left(x \cdot \frac{\log 3}{\log 2}\right)
$$


Since $(f(m, k))$ is almost boundedly invariant we define the sequence of averages $(a(m))$ as above and let

$$
A:=\sup \left\{a(m) \mid m \in \mathbb{N}_{0}\right\}+1 .
$$

Then there exists a positive $d<3$ and a natural number $N_{1}$ such that

$$
\sum_{k=1}^{3^{m-1}}|| f(m, k)|-a(m)|<d^{m} \quad \text { for all } m \geq N_{1} .
$$

Since all elements in the sum which defines $\zeta_{f}(s)$ are non-negative we may rearrange the sum as we please. Further since we are interested in the behaviour of $(x-1) T(x)$ as $x \rightarrow 1+$ any finite number of terms in the sum defining $T(x)$ may be left out, when we prove that $(x-1) T(x)$ is bounded on the interval $(1, \infty)$. We know already by Theorem 3.3 that the function $\zeta_{\mathrm{ZGT}}(x \log 3 / \log 2)$ can be extended to a meromorphic function with a simple pole at 1 . Let us then look at the remaining sum and let us begin to sum for $m \geq N_{1}$. To make the notation easier we will still use the variable $s=x \log 3 / \log 2$ and we get

$$
\begin{aligned}
& 2 \cdot\left(2^{s}-1\right) \zeta(s) \sum_{m=N_{1}}^{\infty} \sum_{k=1}^{3^{m-1}}|f(m, k)| \cdot 2^{-m s} \\
& \quad \leq 2\left(2^{s}-1\right) \zeta(s) \sum_{m=N_{1}}^{\infty} 2^{-m s}\left[3^{m-1} a(m)+\sum_{k=1}^{3^{m-1}}|| f(m, k)|-a(m)|\right] \\
& \quad \leq 2\left(2^{s}-1\right) \zeta(s) \sum_{m=N_{1}}^{\infty} 2^{-m s}\left[3^{m-1} A+d^{m}\right] \\
& \quad=2\left(2^{s}-1\right) \zeta(s)\left[\frac{A}{3}\left(\frac{3}{2^{s}}\right)^{N_{1}} \frac{2^{s}}{2^{s}-3}+\left(\frac{d}{2^{s}}\right)^{N_{1}} \frac{2^{s}}{2^{s}-d}\right] .
\end{aligned}
$$

From here it follows that the function $\zeta_{f}(s) \cdot\left(s-\frac{\log 3}{\log 2}\right)$ is bounded for $s \in\left(\frac{\log 3}{\log 2}, \infty\right)$, and then $T(x)(x-1)$ is bounded on the interval $(1, \infty)$. The lemma then follows from [GBVF], Lemma 7.19 and Lemma 7.20.

Proposition 3.12. Let $(f(m, k))$ be a boundedly almost invariant sequence, with corresponding spectral triple $\mathrm{ST}(f)$, and $\omega$ an ultrafilter on $\mathbb{N}$ which induces a Dixmier trace on the ideal $\mathscr{L}^{(1, \infty)}\left(H_{f}\right)$, then the functional $\varphi$ on $C(\mathrm{SG})$ defined for $g$ in $C(\mathrm{SG})$ by

$$
\varphi(g):=\operatorname{Tr}_{\omega}\left(\left|D_{f}\right|^{-\log 3 / \log 2} \pi_{f}(g)\right)
$$

is a multiple of the Hausdorff integral on the Sierpinski gasket.

Proof. It is well known that the trace property of $\operatorname{Tr}_{\omega}$ implies that $\varphi$ becomes a bounded positive linear functional on $C(\mathrm{SG})$. We show that the measure assigned by $\varphi$ to the portion of Sierpinski gasket which is contained inside or on each $\Delta_{n, j}$ equals 
$3^{-n}$ of the measure assigned to the entire gasket. This will imply that $\varphi$ is a multiple of the Hausdorff integral on the gasket since the $\log 3 / \log 2$-dimensional Hausdorff measure is the unique measure on the gasket satisfying the foregoing scaling property ([Ba]). First of all we will only need to study the Dixmier trace coming from the unbounded Fredholm module $\operatorname{UFM}(f)$, so we will introduce some notations. We will let $\rho_{m, k}$ denote the representation consisting of $|f(m, k)|$ copies of the standard representation of $C\left(\nabla_{m, k}\right)$, and we will let $K_{m, k}$ denote the corresponding Hilbert space. On this space we define $\widetilde{D}_{m, k}$ as the corresponding amplified Dirac operator. Then we define the Hilbert space $K$, the representation $\rho$ and the Dirac operator as the direct sum of these objects over indices $m, k$ with $f(m, k) \neq 0$. By the lemma above $|\widetilde{D}|^{-\log 3 / \log 2}$ is in $\mathscr{L}^{(1, \infty)}$.

Take $F_{n, j}$ to be the triangle $\Delta_{n, j}$ together with its interior. Let us then consider the functions on SG which are the characteristic functions $\chi_{1, j}, j \in\{1,2,3\}$, for the portion of Sierpinski gasket which is contained in $F_{1, j}$. These functions are not continuous on the gasket, but we see that there is a unique way to extend the representation $\rho$ to be defined on these functions too, namely by defining $\rho\left(\chi_{1, j}\right)$ as the orthogonal projection from $K$ onto the subspace $K_{j}$ defined by

$$
K_{j}:=\bigoplus_{\nabla_{m, k} \subset F_{1, j}} K_{m, k} .
$$

To compute $\operatorname{Tr}_{\omega}\left(\rho\left(\chi_{1, j}\right)|\widetilde{D}|^{-\log 3 / \log 2}\right)$, we have to go through summations over decreasing eigenvalues of $|\widetilde{D}|^{-\log 3 / \log 2}$ corresponding to the eigenvectors which are contained in the space $K_{j}$. Here each nonzero eigenvalue for $|\widetilde{D}|^{-\log 3 / \log 2}$ is of the form

$$
3^{-m}|1 / 2+l|^{-\log 3 / \log 2} \text { for } m \in \mathbb{N}, l \in \mathbb{Z} .
$$

The multiplicity of such an eigenvalue is

$$
M(m):=\sum_{\nabla_{m, k} \subseteq \Delta_{1, j}}|f(m, k)| .
$$

In the partial sums used to define the Dixmier trace

$$
\operatorname{Tr}_{\omega}\left(\rho\left(\chi_{1, j}\right)|\tilde{D}|^{-\log 3 / \log 2}\right)
$$

we will now replace the expression

$$
M(m) 3^{-m}|1 / 2+l|^{-\log 3 / \log 2}
$$

by

$$
3^{m-2} a(m) 3^{-m}|1 / 2+l|^{-\log 3 / \log 2},
$$

and then we need a correction term

$$
\left(M(m)-3^{m-2} a(m)\right) 3^{-m}|1 / 2+l|^{-\log 3 / \log 2} .
$$


By checking partial sums of sums corresponding to decreasing eigenvalues, one can se that the sums involving $a(m)$ will be exactly the same as those used to compute $\operatorname{Tr}_{\omega}\left(|\widetilde{D}|^{-\log 3 / \log 2}\right)$, except that they are all scaled by the factor $1 / 3$. For a suitable $d<3$, the sums of the correction terms may be dominated as follows:

$$
\begin{aligned}
\sum_{l \in \mathbb{Z}} \sum_{m \in \mathbb{N}} \mid & M(m)-3^{m-2} a(m)\left|3^{-m}\right| 1 / 2+\left.l\right|^{-\log 3 / \log 2} \\
& =4 \zeta\left(\frac{\log 3}{\log 2}\right) \sum_{m \in \mathbb{N}}\left|M(m)-3^{m-2} a(m)\right| 3^{-m} \\
& \leq 4 \zeta\left(\frac{\log 3}{\log 2}\right) \sum_{m \in \mathbb{N}} 3^{-m} \sum_{\nabla_{m, k} \subseteq \Delta_{1, j}}|| f(m, k)|-a(m)| \\
& \leq 4 \zeta\left(\frac{\log 3}{\log 2}\right) \sum_{m \in \mathbb{N}} 3^{-m} d^{m} \\
& \leq 4 \zeta\left(\frac{\log 3}{\log 2}\right) \frac{d}{3-d} .
\end{aligned}
$$

Because the sum of all these correction terms is finite, the Dixmier trace $\operatorname{Tr}_{\omega}\left(\rho\left(\chi_{1, j}\right)|\widetilde{D}|^{-\log 3 / \log 2}\right)$ is one third of $\operatorname{Tr}_{\omega}\left(|\widetilde{D}|^{-\log 3 / \log 2}\right)$. Repeated use of this argument will show that the measure assigned by $\varphi$ to the portion of the Sierpinski gasket which is contained in $F_{n, j}$ equals $3^{-n}$ of the measure assigned to the entire gasket.

Example 3.13. The boundedly almost invariant sequences do not form a subgroup of $K^{1}(\mathrm{SG})$ : There exist two boundedly almost invariant sequences $f$ and $g$ such that for the sum sequence $h(m, k):=f(m, k)+g(m, k)$, the Dixmier trace associated to $\mathrm{ST}(h)$ is not a multiple of the Hausdorff integral.

Proof. The gasket has the three upward pointing triangles

$$
\Delta_{1, k}, \quad k=1,2,3 .
$$

We let $(f(m, k))$ and $(g(m, k))$ denote the sequences in $\left.K^{1}(\mathrm{SG})\right)$ given by $f(m, k)=$ 1 for all indices $(m, k)$ and

$$
g(m, k)= \begin{cases}-1 & \text { if } \nabla_{m, k} \subset F_{1,1}, \\ 1 & \text { else, }\end{cases}
$$

respectively. Here $F_{1,1}$ is as in the proof of Proposition 3.12. Clearly both are boundedly almost invariant, but we shall see that their sum $h(m, k):=f(m, k)+$ $g(m, k)$ is not. We obtain $a(m)=4 / 3$ for the sequence of averages for $|h(m, k)|$. 
Hence

$$
\begin{aligned}
\limsup _{m \rightarrow \infty}\left(\sum_{k=1}^{3^{m-1}}|| h(m, k)\left|-\frac{4}{3}\right|\right)^{1 / m} & =\limsup _{m \rightarrow \infty}\left(\frac{1}{3} \cdot \frac{4}{3} 3^{m-1}+\frac{2}{3} \cdot \frac{2}{3} 3^{m-1}\right)^{1 / m} \\
& =\limsup _{m \rightarrow \infty}\left(8 \cdot 3^{m-3}\right)^{1 / m}=3,
\end{aligned}
$$

so the sequence $(h(m, k))$ is not boundedly almost invariant.

For any continuous positive function with support inside $F_{1,1}$, the Dixmier trace induced by ST $(h)$ equals that of the Dixmier trace coming from ZGT. This will not be the case, however, for a positive continuous function supported inside $F_{1,2}$. Hence the Dixmier trace corresponding to ST $(h)$ is not a multiple of the Hausdorff integral.

The example shows that in order to obtain a subgroup of sequences $(f(m, k))$ such that the Dixmier trace generated by ST $(f)$ is a multiple of the Hausdorff integral, we can not do with bounded sequences. On the other hand there is a subgroup where all elements behave nicely.

Definition 3.14. The group $c_{1}(\mathrm{HSG}, \mathbb{Z})$ is defined as the set of sequences $(f(m, k))$ such that

$$
\exists t \in \mathbb{Z} \exists M \in \mathbb{N} \forall m \geq M \forall k \in\left\{1, \ldots, 3^{m-1}\right\}: f(m, k)=t .
$$

Proposition 3.15. For $(f(m, k))$ in $c_{1}(\mathrm{HSG}, \mathbb{Z})$ the Dixmier trace associated to the spectral triple $\mathrm{ST}(f)$ is a multiple of the Hausdorff integral.

Proof. The sequences in $c_{1}(\mathrm{HSG}, \mathbb{Z})$ are all boundedly almost invariant.

\section{Aspects of minimality of ZGT}

In [Co3] Connes formulates seven axioms for a spectral triple $(\mathcal{A}, \mathscr{H}, D)$ with $\mathcal{A}$ a commutative algebra. In [Co5] he shows that five of these axioms (in a slightly stronger form) suffice in order to characterize the spectral triples associated to smooth compact manifolds, i.e., from these axioms one can construct a smooth oriented compact $\left(\operatorname{spin}^{c}\right)$ manifold $X$.

The fractal we study is by nature far away from being a smooth manifold, and this is why we can not expect to construct spectral triples for the Sierpinski gasket which satisfy all of Connes' axioms. One of the problems our spectral triples raise is that our algebra of differentiable functions (Lipschitz functions), according to [CIL], Theorem 8.2, is the algebra of functions on the gasket generated by the restrictions of the affine functions. As we remark in that paper, an affine function restricted to a triangle gives a continuous function with constant slopes along the edges. It then 
belongs to the domain of the Dirac operator, but its derivative will not, unless the function is constant. It seems hopeless to get a regularity condition, i.e., to talk about smooth functions in this case. We will therefore stay inside the set-up where we consider spectral triples defined as direct sums of of unbounded Fredholm modules associated to triangles of the form $\nabla_{m, j}$ or $\Delta_{0,1}$. Our main result in this section shows that in this set-up, the geometry, i.e., the representation ([Co3]), of the spectral triple ZGT is the minimal one among those which induce the geodesic distance on the Sierpinki gasket. Recall that the ZGT spectral triple is the direct sum of the unbounded Fredholm modules associated to the big outer triangle and all those associated to the upside down triangles. As the gasket is the closure of the increasing sequence of graphs $G_{n}$ in Figure 2, it is clear that one can leave out the big outer triangle and any finite number of upside down triangles and still gets the gasket as the closure. The corresponding restricted sum of unbounded Fredholm modules will then induce a faithful representation and hence a spectral triple, but the geodesic distance will not be recovered.

We consider the case of a spectral triple which is obtained from ZGT by leaving out one of the triangles. Let us denote such a spectral triple by RGT (reduced gasket triple). We will show:

Proposition 4.1. The metric $d_{\mathrm{RGT}}$ induced by any RGT is equivalent to the geodesic distance $d_{g}$ on the gasket, but it does not coincide with it: There exist points $x, y$ such that

$$
d_{\mathrm{RGT}}(x, y) \geq \frac{3}{2} d_{g}(x, y) .
$$

The rest of this section is devoted to a proof of this proposition divided into several small arguments. A slight modification of the proof of Theorem 8.13 in [CIL] shows that for any spectral triple of the form $\mathrm{ST}(f)$ the metric induced by the distance formula applied to this triple is $d_{g}$. It is well known, see for instance [Ba], that for points $x, y$ on the gasket

$$
\|x-y\| \leq d_{g}(x, y) \leq 8\|x-y\|,
$$

so that the geodesic metric is equivalent to the Euclidean metric.

To enter into the proof of the proposition, let us look at the triple ZGT and suppose that we take out the summand corresponding to the outer triangle $\Delta_{0,1}$. It will become clear that our considerations may be transferred to the situation where we instead remove one of the triangles $\nabla_{m, k}$. In order to clarify things we will give some definitions and figures. Define the graphs

$$
H_{n}:=\bigcup_{m \leq n} \bigcup_{k \leq 3^{m-1}} \nabla_{m, k}, \quad n=1,2, \ldots, \infty .
$$

See the illustration below for a drawing presenting $H_{3}$ and a part of $H_{4}$. 


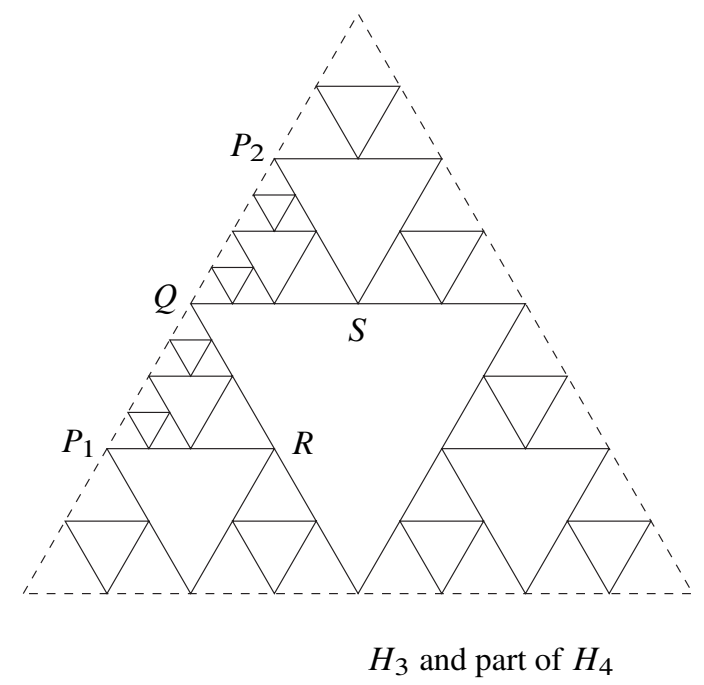

Figure 3

On each graph $H_{n}$ a metric $d_{n}$ is given by the geodesic distance on that graph. As $H_{n} \subseteq H_{n+1}$ the sequence $d_{n+k}(x, y)$ is decreasing for any points $x, y$ from $H_{n}$ as $k \rightarrow \infty$, and we obtain a metric on $H_{\infty}$ by

$$
d_{\infty}(x, y):=\lim _{k \rightarrow \infty} d_{n+k}(x, y) .
$$

Lemma 4.2. For any points $x, y$ in $H_{\infty}$,

$$
d_{g}(x, y) \leq d_{\infty}(x, y) \leq 2 d_{g}(x, y) .
$$

Proof. Fix $n$ such that $x, y \in H_{n}$. It is known from the analysis in [CIL], see Lemma 8.11 and the following remarks, that a shortest path from $x$ to $y$ can be found along the edges of $G_{n}=H_{n} \cup \Delta_{0,1}$. As any piece of this path going through $\Delta_{0,1}$ can be replaced by two pieces of the same length in $H_{n}$, we have

$$
d_{g}(x, y) \leq d_{\infty}(x, y) \leq d_{n}(x, y) \leq 2 d_{g}(x, y) .
$$

Since the set of points in $H_{\infty}$ is dense in the gasket, it is possible to extend $d_{\infty}$ by continuity to a metric $d_{0}$ on the gasket, and we have:

Corollary 4.3. For any pair of points $x, y$ on the Sierpinski gasket,

$$
d_{g}(x, y) \leq d_{0}(x, y) \leq 2 d_{g}(x, y)
$$

so that the metric $d_{0}$ is equivalent to the geodesic distance. 
We will show next that $d_{0}$ does not coincide with the geodesic distance. As mentioned above, let RGT denote the reduced spectral triple obtained as the direct sum of all the unbounded Fredholm modules associated to all the triangles $\nabla_{m, j}$ (thus leaving out $\Delta_{0,1}$ ), and let $D_{\mathrm{RGT}}, \pi_{\mathrm{RGT}}$ be the corresponding Dirac operator and representation. We consider two vertices $P_{1}$ and $P_{2}$ on $\Delta_{0,1}$ as sketched in Figure 3 and shall show that

$$
d_{n}\left(P_{1}, P_{2}\right)=\frac{3}{2} d_{g}\left(P_{1}, P_{2}\right) \text { for } n \geq 3
$$

This implies that

$$
d_{0}\left(P_{1}, P_{2}\right)=\frac{3}{2} d_{g}\left(P_{1}, P_{2}\right) .
$$

We first observe that the function $d: \mathrm{SG} \rightarrow \mathbb{R}_{+} \cup\{0\}$ defined by $d(x):=d_{0}\left(P_{1}, x\right)$ satisfies

$$
\left\|\left[D_{\mathrm{RGT}}, \pi_{\mathrm{RGT}}(d)\right]\right\|=1,
$$

and this follows as in the proof of [CIL], Lemma 8.12, noting that on any edge in $H_{n}$ the function $g$ is differentiable except at at most one point and the numerical value of the derivative, when defined, is bounded by 1 . Hence

$$
d_{\mathrm{RGT}}\left(P_{1}, x\right) \geq d_{0}\left(P_{1}, x\right) .
$$

We consider $d$ on the edge of $\Delta_{0,1}$ which contains $P_{1}$ and $P_{2}$ and abbreviate $\alpha=$ $d_{g}\left(P_{1}, P_{2}\right)$. Clearly any shortest path from $P_{1}$ to $P_{2}$ will pass through $Q$ and

$$
d_{2}\left(P_{1}, P_{2}\right)=2 \alpha, \quad d_{3}\left(P_{1}, P_{2}\right)=\frac{3}{2} \alpha .
$$

Next, considering the triangles with vertices $P_{1}, R, Q$ and $Q, S, P_{2}$ we see that determining a shortest path in $H_{4}$ is analogous to determining a shortest path in $\mathrm{H}_{3}$ by scaling, and $d_{4}\left(P_{1}, P_{2}\right)=\frac{3}{2} \alpha$. Iteration then shows that

$$
d_{n}\left(P_{1}, P_{2}\right)=\frac{3}{2} \alpha \text { for all } n \geq 3 .
$$

Taking the limit as $n \rightarrow \infty$ we obtain (3) and, in view of (4), inequality (2) in Proposition 4.1.

Comment 4.4. Proposition 4.1 above shows that no summand in our spectral triple ZGT can be left out, if the geodesic distance shall be obtained via Connes' formula. On the other hand one may leave out any finite number of summands and still get a spectral triple, because the union of the remaining triangles $\nabla_{m, j}$ is dense in the gasket. Although such a spectral triple will not reproduce the geodesic distance, the volume form will still be the one obtained from ZGT. 


\section{The Sierpinski pyramid: constructions, K-theory and K-homology}

The Sierpinski pyramid is the 3-dimensional version of the Sierpinski gasket. One starts, for example, with a solid regular tetrahedron $P_{0}$ (Figure below on the left) and divides it into eight identical regular tetrahedra. One then cuts out all the smaller tetrahedra except for the ones at the vertices of the starting tetrahedron, obtaining $P_{1}$ (Figure below on the right).
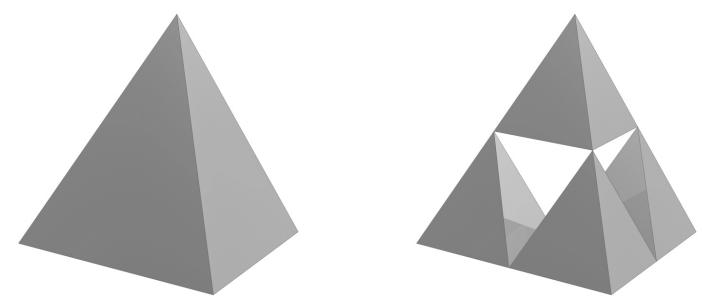

Figure 4

One iterates this procedure (see below the illustration of $P_{2}$ ).

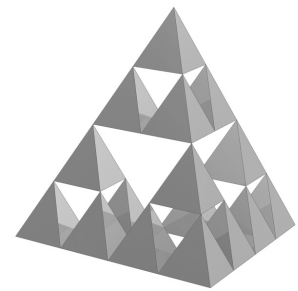

Figure 5

(The drawings are done by Jürgen Meier, www.3d-meier.de.)

In the $n$-th step, one cuts away $4 \cdot 4^{n-1}$ regular tetrahedra, or $4^{n-1}$ regular octahedra, with side length equal to $2^{-n}$ of that of the original one. The Sierpinski pyramid SP is then the limit of this decreasing sequence of compact subsets $P_{n}$, of $P_{0}$. For any $n \in \mathbb{N}, P_{n}$ is the union of $4^{n}$ solid tetrahedra, say $P_{n, k}, 1 \leq k \leq 4^{n}$, with the side length $2^{-n}$ of that of $P_{0}$. One calculates easily the Hausdorff dimension of this fractal. Indeed the Sierpinski pyramid is a self-similar set satisfying the open set condition and can be constructed out of four similarities of ratio $1 / 2$. This implies that its Hausdorff dimension $s$ is the solution of the equation $4 \cdot(1 / 2)^{s}=1$ and thus $s=2$. The $\mathrm{C}^{*}$-algebra of the continuous functions on the Sierpinski pyramid $C(\mathrm{SP})$ is the direct limit of $\mathrm{C}^{*}$-algebras $C\left(P_{n}\right)$ and the homomorphisms given by the restriction maps. We will use this picture to compute $K_{1}(C(\mathrm{SP}))$. We start by considering $P_{1}$. If we retract each $P_{1, k}, 1 \leq k \leq 4$, along oblique edges, as shown below, we obtain from $P_{1}$ the graph below, which is homotopic to the three-leaved 

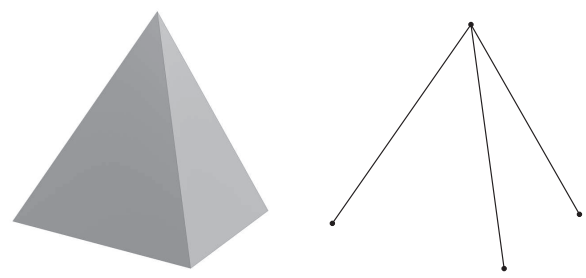

Figure 6

rose $R_{3}$ (recall that every finite (connected) graph is homotopic to a $n$-leaved rose, where $n$ is the number of edges not belonging to a maximal tree of the graph; see, for example, $[\mathrm{AB}])$.
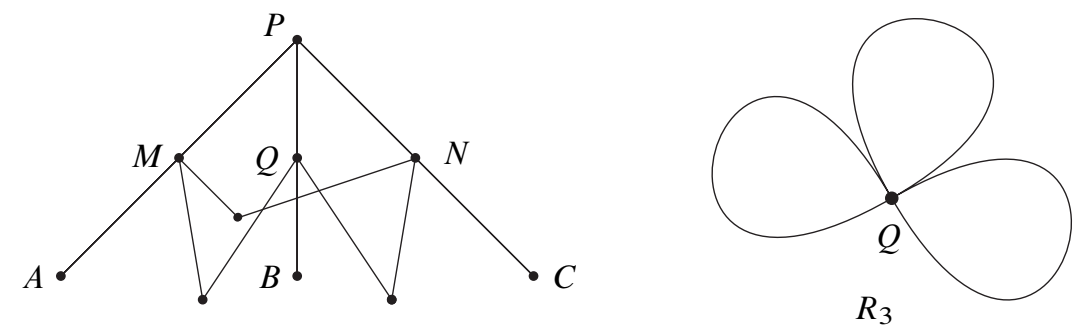

Figure 7

The three leaves correspond to the holes in the oblique faces of the initial tetrahedron. In the subsequent steps, for any $n \geq 2$, the cutting of an octahedron from one of the tetrahedra of $P_{n-1}$ will produce each time three holes in the oblique faces of $P_{n-1}$. Hence $P_{n}$ will be homotopic to a rose having as many leaves as one counts holes in every oblique face of any of the pyramids at the $n$-step in the construction of SP, i.e.,

$$
3+4 \cdot 3+\cdots+4^{n-1} \cdot 3=4^{n}-1
$$

leaves. The K-theory of the $n$-leaved rose $R_{n}$ is easily computed using a six-term exact sequence and it follows from [RLL], p. 232, that

$$
K_{0}\left(C\left(R_{n}\right)\right)=\mathbb{Z} \quad \text { and } \quad K_{1}\left(C\left(R_{n}\right)\right)=\mathbb{Z}^{n}
$$

The singular homology of the $n$-leaved rose is given by $H^{1}\left(R_{n}\right)=\mathbb{Z}^{n}([\mathrm{AB}],[\mathrm{Mu}])$. We thus conclude

$$
K_{1}\left(C\left(P_{n}\right)\right)=H^{1}\left(P_{n}\right)=\mathbb{Z}^{4^{n}-1}
$$

As the direct limit of $K_{1}\left(C\left(P_{n}\right)\right), K_{1}(C(\mathrm{SP}))$ will count the holes in every oblique face of any of the small pyramids that arise in the construction of the Sierpinski 
pyramid, ${ }^{1}$ hence

$$
K_{1}(C(\mathrm{SP}))=\bigoplus_{n=1}^{\infty} \mathbb{Z}^{3 \cdot 4^{n-1}} .
$$

We shall remark here that also for the Sierpinski pyramid

$$
K_{1}(C(\mathrm{SP})) \cong \check{H}^{1}(\mathrm{SP}) ;
$$

in fact, since SP is the intersection of the $P_{n}$, we obtain from [Mu], Theorem 73.4,

$$
\breve{H}^{1}(\mathrm{SP})=\lim _{\longrightarrow} H^{1}\left(P_{n}\right) \quad \text { and } \quad H^{1}\left(P_{n}\right)=K_{1}\left(C\left(P_{n}\right)\right) .
$$

Let $X$ be a compact subset of the space $\mathbb{R}^{3}$ and $u$ a unitary in some matrix algebra $M_{k}(C(X))$, i.e., $u \in U_{k}(C(X))$. Then the $K_{1}$ class $[u]$ of $u$ is not in general represented by a unitary $v$ in $C(X)$, but the above analysis shows that for the Sierpinski pyramid this is the case. In an unpublished article [Br] (see [BDF], [Do]) Larry Brown has extended the result in $[\mathrm{BDF}]$ to $\mathbb{R}^{3}$, i.e., he showed that the index map (in Kasparov's picture)

$$
K^{1}(C(X)) \ni[(H(\pi), F)] \mapsto \Phi_{(H, F)} \in \operatorname{Hom}\left(K_{1}(C(X)), \mathbb{Z}\right)
$$

defined by

$$
\Phi_{(H, F)}([u])=-\operatorname{Index}\left(P_{k} \pi_{k}(u) P_{k}\right), \quad u \in U_{k}(C(X)), k \in \mathbb{N},
$$

is an isomorphism for any compact $X \subset \mathbb{R}^{3}$.

For the Sierpinski pyramid we conclude that

$$
\Phi_{(H, F)}([u])=-\operatorname{Index}(P \pi(u) P), \quad u \in U(C(X)) .
$$

\section{A family of spectral triples that generates $K^{1}(C(\mathrm{SP}))$}

We suppose that the initial solid tetrahedron $P_{0}$ is placed such that one vertex is pointing upwards (as in Figure 4 ) and has the side length $2 \pi / 3$. The four equilateral triangles of perimeter $2 \pi$ which are the boundaries of the four faces of $P_{0}$ are denoted by $\Delta_{0, k}, k \in\{1, \ldots, 4\}$.

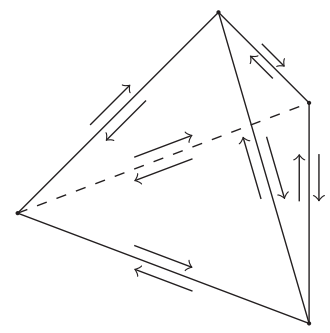

Figure 8

\footnotetext{
${ }^{1}$ This result was obtained in the diploma thesis [Ha] of Stefan Hasselmann supervised by C. Ivan.
} 
Numbering 6.1. By $\Delta_{1,1, k}, k \in\{1, \ldots, 8\}$, we denote the eight equilateral triangles of perimeter $\pi$ which are the boundaries of the faces of the cut out octahedron in the first step of the construction procedure of the Sierpinski pyramid. We will consider them numbered as follows:

(1) $\Delta_{1,1, k}, k \in\{1,2,3\}$, for the boundaries of the holes into an oblique face of $P_{0}$ generated by cutting out the octahedron.

(2) $\Delta_{1,1,4}$ for the boundary of the hole into the horizontal face of $P_{0}$, and

(3) $\Delta_{1,1, k}, k \in\{5, \ldots, 8\}$, for the boundaries of the remaining faces of the cut out octahedron.
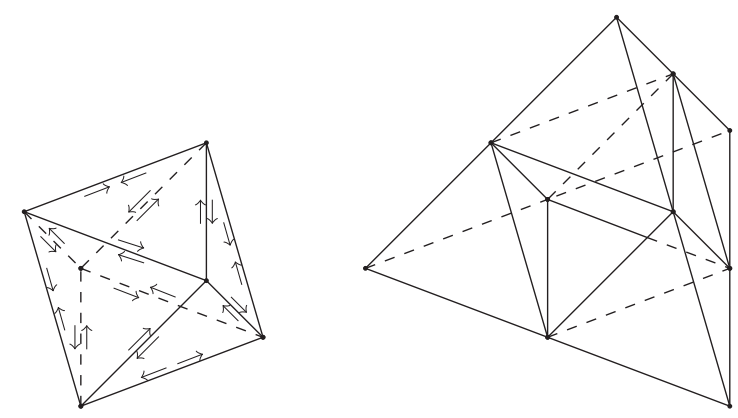

Figure 9

In general, for any $n \in \mathbb{N}$, we introduce a numbering of the $8 \cdot 4^{n-1}$ equilateral triangles of perimeter $2^{1-n} \pi$ which are the boundaries of the faces of all cut out octahedra in the $n$-th step of the construction of the Sierpinski pyramid and denote the numbered triangles by $\Delta_{n, m, k}, m \in\left\{1, \ldots, 4^{n-1}\right\}, k \in\{1, \ldots, 8\}$. The numbering of the triangles obeys the following rules: for any $m \in\left\{1, \ldots, 4^{n-1}\right\}$,

(1) $\Delta_{n, m, k}, k=1,2,3$, for the boundaries of the holes in an oblique face of $P_{n-1, m}$,

(2) $\Delta_{n, m, 4}$ for the boundary of the hole in the horizontal face of $P_{n-1, m}$, and

(3) $\Delta_{n, m, k}, k=5, \ldots, 8$, for the boundaries of the remaining faces of the octahedron which is cut out of $P_{n-1, m}$.

We will construct unbounded Fredholm modules over the algebra $C(\mathrm{SP})$ based on the triangles $\Delta_{n, m, k}$ similarly as for the Sierpinski gasket. We endow each face of any pyramid with the orientation induced by the outer normal vector and orient the boundary correspondingly. With this orientation we construct an unbounded Fredholm module $\operatorname{UFM}\left(\Delta_{0, k}\right)$ over $C(\mathrm{SP})$ in the same way we did for the initial triangle $\Delta_{0,1}$ for the Sierpinski gasket. For $n \geq 1$ and $m$ fixed in $\left\{1, \ldots, 4^{n-1}\right\}$ the triangles $\Delta_{n, m, k}, 1 \leq k \leq 8$, are all boundaries of a face of an octahedron. Then for each set of indices $(n, m, k)$ we can construct an unbounded Fredholm module $\operatorname{UFM}\left(\Delta_{n, m, k}\right)$ for $C(\mathrm{SP})$. By reversing the orientation in the parameterization of the 
triangles we obtain the unbounded Fredholm modules denoted by $\overline{\operatorname{UFM}\left(\Delta_{n, m, k}\right)}$.

To obtain a family of spectral triples which would encode as well as possible the fractal geometry of the pyramid and generate its $K^{1}$-group we use the same idea as for the Sierpinksi gasket. We first construct a spectral triple which encodes the fractal geometry of the pyramid and induces the 0-index map, and thus the 0element of $K^{1}(C(\mathrm{SP}))$. To this spectral triple we then add (the appropriate number of) circle spectral triples associated to the boundary of each hole (with in the appropriate orientation) in each oblique face of any small pyramid arising in the construction of the Sierpinski pyramid.

A spectral triple which encodes the fractal geometry of the Sierpinski pyramid and induces the 0 -index map is given in the following definition.

Definition 6.2. The direct sum of unbounded Fredholm modules for the Sierpinski pyramid given by

$$
\bigoplus_{k=1}^{4} \operatorname{UFM}\left(\Delta_{0, k}\right) \oplus \bigoplus_{n=1}^{\infty} \bigoplus_{m=1}^{4^{n-1}} \bigoplus_{k=1}^{8} \operatorname{UFM}\left(\Delta_{n, m, k}\right)
$$

is a spectral triple, which is denoted by ZPT (the zero pyramid triple). The Hilbert space and the Dirac operator of this spectral triple are denoted by $H_{\mathrm{ZPT}}$ and $D_{\mathrm{ZPT}}$, respectively.

Theorem 6.3. The bounded Fredholm module coming from the polar decomposition of $D_{\mathrm{ZPT}}$ induces the trivial element of the group $K^{1}(C(\mathrm{SP}))$.

The spectral triple ZPT has the following geometric properties:

(i) The metric induced is the geodesic distance.

(ii) The ZPT is summable for any positive $s>2$. Its zeta-function $\zeta_{\mathrm{ZPT}}(s)$ is meromorphic with a simple pole at 2 and is given by $\zeta_{\mathrm{ZPT}}(s)=8 \cdot \frac{\left(2^{s}-1\right)\left(2^{s}-2\right)}{2^{s}-4} \zeta(s)$.

(iii) Let $\mu$ denote the normalized 2-dimensional Hausdorffmeasure on the Sierpinski pyramid. Then for any Dixmier trace and any continuous function $g$ in $C(\mathrm{SP})$ we have

$$
\operatorname{Tr}_{\omega}\left(\left|D_{\mathrm{ZPT}}\right|^{-2} \pi_{\mathrm{ZPT}}(g)\right)=\frac{6}{\log 2} \cdot \zeta(2) \cdot \int_{\mathrm{SP}} g(x) d \mu(x) .
$$

Proof. Using similar arguments as in [CIL], pp. 27-28, we can show that the direct sum is a spectral triple (this result and (i) were also obtained in [Ha]).

Let $u$ be a unitary of $C(\mathrm{SP})$. We refer to Proposition 3.1 (i) to write the Khomology element induced by ZPT:

$$
\Phi_{\mathrm{ZPT}}([u])=\sum_{k=1}^{4} w_{\Delta_{0, k}}(u)+\sum_{n=1}^{\infty} \sum_{m=1}^{4^{n-1}} \sum_{k=1}^{8} w_{\Delta_{n, m, k}}(u) .
$$


Since from a certain number $n_{0}$ on the winding number of $u$ around any $\Delta_{n, m, k}$, $n>n_{0}, m=1, \ldots, 4^{n-1}, k=1, \ldots, 8$, vanishes, the sum is finite. Let us compute it. We notice that $\sum_{k=1}^{4} w_{\Delta_{0, k}}(u)=0$ since each edge of the starting tetrahedron is covered twice but in opposite directions and thus the sum of the winding numbers of $u$ around the four triangles $\Delta_{0, k}$ is 0 , see Figure 8. In fact for any $n \in\left\{1, \ldots, n_{0}\right\}$ and $m \in\left\{1, \ldots 4^{n-1}\right\}$ the expression $\sum_{k=1}^{8} w_{\Delta_{n, m, k}}(u)$ is 0 since each edge in the octahedron numbered $(n, m)$ will be counted twice and in opposite directions, see Figure 9. Thus the sum of the winding numbers of $u$ around the eight triangles is 0 . In conclusion the corresponding element in $K^{1}(C(\mathrm{SP}))$ of ZPT is trivial. We now fix a positive number $s>2$ and compute the zeta-function of ZPT in $s$. We remark first that, for any $n \in \mathbb{N}_{0}$, the zeta-function in a point $s>2$ for $\Delta_{0, k}, k=1, \ldots$, 4 , and $\Delta_{n, m, k}, n \in \mathbb{N}, m=1, \ldots, 4^{n-1}, k=1, \ldots, 8$, is the zeta-function in $s$ for $\Delta_{n, j}$ from the Sierpinski gasket case, and thus equals $2 \cdot 2^{-n s} \cdot\left(2^{s}-1\right) \cdot \zeta(s)$, see the equation (1). There are four triangles $\Delta_{0, k}$ and $8 \cdot 4^{n-1}$ triangles $\Delta_{n, m, k}$. Hence the zeta-function for ZPT in a point $s>2$ is

$$
\begin{aligned}
\zeta_{\mathrm{ZPT}}(s) & =4 \cdot 2\left(2^{s}-1\right) \cdot \zeta(s)+\sum_{n=1}^{\infty} 8 \cdot 4^{n-1} \cdot 2 \cdot 2^{-n s}\left(2^{s}-1\right) \cdot \zeta(s) \\
& =8 \cdot\left(2^{s}-1\right) \zeta(s)+8 \cdot 2^{1-s} \cdot\left(2^{s}-1\right) \frac{1}{1-\left(4 / 2^{s}\right)} \cdot \zeta(s) \\
& =8 \cdot\left(2^{s}-1\right) \zeta(s)\left(1+2^{1-s} \cdot \frac{1}{1-4 / 2^{s}}\right) \\
& =8 \cdot \frac{\left(2^{s}-1\right)\left(2^{s}-2\right)}{2^{s}-4} \zeta(s) .
\end{aligned}
$$

Moreover, we obtain

$$
\begin{aligned}
\operatorname{Tr}_{\omega}\left(\left|D_{\mathrm{ZPT}}\right|^{-2}\right) & =\lim _{x \rightarrow 1+}(x-1) \zeta_{\mathrm{ZPT}}(x \cdot 2) \\
& =4 \cdot 3 \zeta(2) \lim _{x \rightarrow 1+}(x-1) \frac{1}{4^{x-1}-1}=\frac{6}{\log 2} \cdot \zeta(2) .
\end{aligned}
$$

In accordance with the convention in Numbering 6.1 we shall index the nonhorizontal holes of Sierpinski pyramid by

$$
\mathrm{HSP}:=\left\{(n, m, k) \mid n \in \mathbb{N}, m=1, \ldots, 4^{n-1}, k=1,2,3\right\},
$$

and thus we may write

$$
\begin{aligned}
& K_{1}(C(\mathrm{SP}))=\bigoplus_{(n, m, k) \in \mathrm{HSP}} \mathbb{Z}, \\
& K^{1}(C(\mathrm{SP}))=\operatorname{Hom}\left(K_{1}(C(\mathrm{SP})), \mathbb{Z}\right)=\prod_{(n, m, k) \in \mathrm{HSP}} \mathbb{Z} .
\end{aligned}
$$

We shall now construct a spectral triple which induces any prescribed element in the group $K^{1}(C(\mathrm{SP}))$. 
Theorem 6.4. Let $(f(n, m, k))_{n, m, k} \in \prod_{(n, m, k) \in \mathrm{HSP}} \mathbb{Z}$. Then

$$
\mathrm{ST}(f):=\mathrm{ZPT} \oplus \operatorname{UFM}(f),
$$

where $\operatorname{UFM}(f)$ denotes the direct sum

$$
\bigoplus_{\substack{(n, m, k) \in \mathrm{HSP}: \\ f(n, m, k) \neq 0}} \begin{cases}\operatorname{UFM}\left(\Delta_{n, m, k}\right) \stackrel{f(n, m, k)}{\oplus \cdots \oplus} \operatorname{UFM}\left(\Delta_{n, m, k}\right) & \text { if } f(n, m, k)>0, \\ \overline{\operatorname{UFM}\left(\Delta_{n, m, k}\right)} \stackrel{-f(n, m, k)}{\oplus \cdots \oplus} \overline{\operatorname{UFM}\left(\Delta_{n, m, k}\right)} & \text { if } f(n, m, k)<0,\end{cases}
$$

is a spectral triple which induces the geodesic distance on the pyramid and the $K^{1}$ element $(f(n, m, k))_{n, m, k}$.

As for the gasket it is easily seen that if a function $(f(n, m, k))$ is bounded then the summability properties for $\mathrm{ST}(f)$ are the same as for ZPT. It is also possible to check that if a function $(f(n, m, k))$ has the property that it is constant for all indices with $n \geq N$ for some natural number $N$ then the volume form will be proportional to that of the 2-dimensional Hausdorff measure.

At last we will examine the possibility to have an unbounded function $f(n, m, k)$ such that the triple $\mathrm{ST}(f)$ is summable for any $p>2$. We write down - at least formally - the zeta-function $\zeta_{f}(s)$ for $\operatorname{ST}(f)$, and we obtain

$$
\begin{aligned}
\zeta_{f}(s) & =\zeta_{\mathrm{ZPT}}(s)+\sum_{n=1}^{\infty} \sum_{m=1}^{4^{n-1}} \sum_{k=1}^{3}|f(n, m, k)|\left(2 \cdot 2^{-n s} \cdot\left(2^{s}-1\right) \cdot \zeta(s)\right) \\
& =\zeta_{\mathrm{ZPT}}(s)+2 \cdot\left(2^{s}-1\right) \zeta(s) \sum_{n=1}^{\infty}\left(\sum_{m=1}^{4^{n-1}} \sum_{k=1}^{3}|f(n, m, k)|\right) \cdot 2^{-n s} .
\end{aligned}
$$

Based on the root criterion we then get:

Theorem 6.5. Let $(f(n, m, k)) \in \prod_{\mathrm{HSP}} \mathbb{Z}$ then $\mathrm{ST}(f)$ is summable for any $p>2$ if

$$
\limsup _{n \rightarrow \infty}\left(\sum_{m=1}^{4^{n-1}} \sum_{k=1}^{3}|f(n, m, k)|\right)^{1 / n} \leq 4 .
$$

\section{References}

[AB] E. Artin and H. Braun, Introduction to algebraic topology. Charles E. Merrill Publishing Co., Columbus, Ohio 1969. Zbl 0181.51201 MR 0247624

[BJ] S. Baaj and P. Julg, Théorie bivariante de Kasparov et opérateurs non bornés dans les $C^{*}$-modules hilbertiens. C. R. Acad. Sci. Paris Sér. I Math. 296 (1983), 875-878. Zbl 0551.46041 MR 715325 
[Ba] M. T. Barlow, Diffusions on fractals. In Lectures on probability theory and statistics (Saint-Flour, 1995), Lecture Notes in Math. 1690, Springer, Berlin 1998, 1-121. Zbl 0916.60069 MR 1668115

[Br] L. G. Brown, The topology of the group $\operatorname{Ext}(X)$. Unpublished.

[BDF] L. G. Brown, R. G. Douglas, and P. A. Fillmore, Extensions of $C^{*}$-algebras and K-homology. Ann. of Math. (2) 105 (1977), 265-324. Zbl 0376.46036 MR 0458196

[CI] E. Christensen and C. Ivan, Spectral triples for AF $C^{*}$-algebras and metrics on the Cantor set. J. Operator Theory 56 (2006), 17-46. Zbl 1111.46052 MR 2261610

[CIL] E. Christensen, C. Ivan, and M. L. Lapidus, Dirac operators and spectral triples for some fractal sets built on curves. Adv. Math. 217 (2008), 42-78. Zbl 1133.28002 MR 2357322

[Co1] A. Connes, Non-commutative differential geometry. Inst. Hautes Études Sci. Publ. Math. 62 (1985), 41-144. Zbl 0592.46056 MR 0823176

[Co2] A. Connes, Noncommutative geometry. Academic Press, San Diego, CA, 1994. Zbl 0818.46076 MR 1303779

[Co3] A. Connes, Gravity coupled with matter and the foundation of non-commutative geometry. Comm. Math. Phys. 182 (1996), 155-176. Zbl 0881.58009 MR 1441908

[Co4] A. Connes, Unpublished notes on a Dirac operator associated to the Cantor subset of the unit interval. Electronic message to Michel Lapidus, May 2002.

[Co5] A. Connes, On the spectral characterisation of manifolds. To appear in J. Noncommut. Geom. arXiv:0810.2088

[Do] R. G. Douglas, $C^{*}$-algebra extensions and K-homology. Ann. of Math. Stud. 95, Princeton University Press, Princeton, N.J., 1980 Zbl 0458.46049 MR 571362

[GBVF] J. M. Gracia-Bondía, J. C. Várilly, and H. Figueroa, Elements of noncommutative geometry. Birkhäuser Adv. Texts, Birkhäuser, Boston 2001. Zbl 0958.46039 MR 1789831

[Ha] S. Hasselmann, Geometrische und topologische Untersuchungen der SierpinskiPyramide mit Methoden der Nichtkommutativen Geometrie. Diplomarbeit, Leibniz Universität Hannover, Hannover 2009.

[HR] N. Higson and J. Roe, Analytic K-homology. Oxford Math. Monogr., Oxford University Press, Oxford 2000. Zbl 0968.46058 MR 1817560

[Ka1] G. G. Kasparov, Topological invariants of elliptic operators. I: $K$-homology. $I z v$. Akad. Nauk SSSR Ser. Mat. 39 (1975), 796-838; English transl. Math. USSR-Izv. 9 (1975), 751-792. Zbl 0337.58006 MR 0488027

[Ka2] G. G. Kasparov, The $K$-functor in the theory of extensions of $C^{*}$-algebras. Funktsional. Anal. i Prilozhen. 13 (1979), 73-74; English transl. Funct. Anal. Appl. 13 (1980), 296-297. Zbl 0447.46053 MR 554420

[Ka3] G. G. Kasparov, The operator $K$-functor and extensions of $C^{*}$-algebras. Izv. Akad. Nauk SSSR Ser. Mat. 44 (1980), 571-636; English transl. Math. USSR-Izv. 16 (1981), 513-572. Zbl 0464.46054 MR 582160

[La1] M. L. Lapidus, Analysis on fractals, Laplacians on self-similar sets, noncommutative geometry and spectral dimensions. Topol. Methods Nonlinear Anal. 4 (1994), 137-195. Zbl 0836.35108 MR 1321811 
[La2] M. L. Lapidus, Towards a noncommutative fractal geometry? Laplacians and volume measures on fractals. In Harmonic analysis and nonlinear differential equations (Riverside, CA, 1995), Contemp. Math. 208, Amer. Math. Soc., Providence, RI, 1997, 211-252. Zbl 0889.58012 MR 1467009

$[\mathrm{Mu}] \quad$ J. R. Munkres, Elements of algebraic topology. Addison-Wesley Publishing Company, Redwood City, Calif., 1984. Zbl 0673.55001 MR 755006

[RLL] M. Rørdam, F. Larsen, and N. Laustsen, An introduction to $K$-theory for $C^{*}$-algebras. London Math. Soc. Stud. Texts 49, Cambridge University Press, Cambridge 2000. Zbl 0967.19001 MR 1783408

Received March 10, 2010

E. Christensen, Department of Mathematics, University of Copenhagen, 2100 Copenhagen, Denmark

E-mail: echris@math.ku.dk

C. Ivan, Center for RNA Interference and Non-Coding RNA's, The University of Texas, M.D. Anderson Cancer Center, Smith Research Building, Houston, Texas 77054, U.S.A.

E-mail: civan@mdanderson.org

E. Schrohe, Institut für Analysis, Leibniz Universität Hannover, 30167 Hannover, Germany

E-mail: schrohe@math.uni-hannover.de 